\title{
REDE DE COOPERAÇÃO TECNOLÓGICA DA PETROBRAS E UNIVERSIDADES E DAS SUAS ÁREAS DE TECNOLOGIA
}

\author{
Carolina da Silveira Bueno*; José Maria Ferreira Jardim da Silveira*; Antonio Marcio Buainain * \\ Maria Ester Soares Dal Poz ${ }^{* *}$ \\ *Núcleo de Economia Agrícola, Instituto de Economia - Universidade Estadual de Campinas \\ ** Faculdade de Ciências Aplicadas - Universidade Estadual de Campinas
}

\begin{abstract}
This paper aims to analyze Petrobras' technology cooperation network with Brazilian universities. The study focus on two main issues. The first consists of an evaluation of the technological areas in which Petrobras is working and the second question explores Petrobras university-level relations in terms of technology areas. The analysis is based on data extracted from the Derwent Innovation Index database. Ten universities were identified in the network owned by Petrobras. The technological areas range from technologies for extraction and production of oil and also the development of new materials such as plastic and oil for printers. In addition, in order to evaluate the state's intellectual property regime, a third source was collected from technical visits held at Petrobras' CENPES.
\end{abstract}

Key Words: Collaborative Network; Innovation; Patents; Petrobras; IPC.

\section{Resumo}

Este artigo tem por objetivo analisar a rede de cooperação tecnológica da Petrobras com as universidades brasileiras. O estudo centra-se em duas questões principais. A primeira consiste em uma avaliação das áreas tecnológicas em que a Petrobras está atuando e a segunda questão destina-se a explorar as relações universidade-Petrobras em termos de áreas de tecnologia. A análise é baseada em dados extraídos de patentes da base de dados da Derwent Innovation Index. Dez universidades foram identificadas na rede de titularidade com a Petrobras. As áreas tecnológicas estão desde tecnologias para extração e produção de petróleo e também do desenvolvimento de novos materiais, como plástico e óleo para impressoras. Além disso, a fim de avaliar o regime de propriedade intelectual da estatal, uma terceira fonte foi recolhida a partir de visitas técnicas realizada no CENPES da Petrobras.

Palavras-chave: Rede de colaboração; Inovação; Patentes; Petrobras; IPC.

JEL Classification Numbers: O30, O31.

ABEIN - Área 4.4.

\section{INTRODUÇÃO}

O presente estudo introduz o cenário de complexidade que envolve o regime tecnológico aos quais os processos inovativos estão ligados. A complexidade, segundo Frenken (2006), está justamente em mostrar a impossibilidade de implementar sistemas de regulação das novas tecnologias sem que isto determine a necessidade de modificações que implicam investimentos e relações intersetoriais por parte das distintas unidades de pesquisa. As modificações nos processos inovativos são observadas entre a Empresa Brasileira de Petróleo (Petrobras) e as universidades brasileiras e das tecnologias envolvidas em suas redes tecnológicas. A metodologia utiliza patentes da base da Derwent como insumo para investigar a co-autoria nas patentes e as áreas tecnológicas de pesquisa. As informações sobre o regime de propriedade intelectual da Petrobras foram coletadas durante as visitas técnicas realizada no CENPES/Petrobras (Centro de Pesquisas da Petrobras), localizada na cidade do Rio de Janeiro, no período de 2014 e 2016. Este artigo é parte dos resultados de pesquisa referente ao projeto da FAPESP. "Innovation for a bio based economy in Brazil: challenges and hurdles in technology development for sustainable and economic utilization of biomass". Nesse sentido, as informações utilizadas neste trabalho são resultado de uma articulação institucional muito mais ampla e muito profícua que envolveu, durante 2 anos, várias pessoas do CENPES: i) Gerência de Biotecnologia: Lídia Maria Melo Santa Anna; Absai da Conceição Gomes; Juliana Vaz Bevilaqua; ii) Gerência de Propriedade Intelectual: Bernadete Rulff; iii) 
Gerência PDEDS/AMA: Suzana Sattamini e Eduardo Barcelos Platte. Por fim, o trabalho tem por objetivo mostrar como os complexos arranjos colaborativos entre as instituições de pesquisa resultam em desenvolvimento de inovações.

\subsection{Aspectos conceituais metodológicos}

Um trabalho cujo objetivo é gerar dados primários a respeito das quais se tem pouca ou nenhuma informação envolve uma série de definições. Em primeiro lugar, é preciso definir os conceitos utilizados, já que o objeto de análise é novo (nesse campo da economia da inovação).

Redes aqui são representadas por grafos, que se traduzem numa ilustração da rede representada da seguinte maneira: um grafo direcionado consiste de um conjunto $\mathrm{V}$ de vértices (ou nós), um conjunto $\mathrm{E}$ de arestas (links ou Edges), $\mathrm{E} \rightarrow \mathrm{V}$, onde (e) é a fonte e (v) é o alvo da aresta direcionada (Goyal, 2007). Então, qualquer conjunto de elementos de nós no qual alguns deles estão conectados através de links será representado. Aqui a rede é gerada pela titularidade de patentes em que a Petrobras e as universidades são representadas pelos vértices, onde a Petrobras é a fonte e o alvo da aresta são as universidades. Ou seja, somente as patentes de co-autoria entre Petrobras e universidades estará representada na rede.

Rede de cooperação tecnológica aqui é definida como sendo a relação de duas ou mais instituições que tem titularidade numa mesma patente. Essa relação não é analisada manualmente, e sim, através de um programa de computador (The VantagePoint). Além disso, o programa cria uma matriz de adjacência cuja função é mostrar a relação entre dois elementos ou mais, um chamado de referência e o outro de vizinho. Neste caso, a referência estará representada pela Petrobras. Este procedimento permite que sejam identificadas as áreas de especialização e o os agentes (neste caso as universidades) conectados na rede.

Área de especialização tecnológica aqui é definida como sendo a área do conhecimento que os agentes estão operando. Ou seja, se é na área de química, de engenharia, e assim por diante. Essa análise é feita através da análise de IPCs (Classificação Internacional de Patentes) a partir da análise da matriz (obrigatoriamente quadrada) de coocorrência, que representa em cada elemento o número de vezes que ocorreu uma transição do vizinho considerando a distância e a direção. Com isto é possível identificar as tecnologias das patentes. Então, sabendo disso, pode-se averiguar as áreas tecnológicas. A base de dados escolhida para levantar informações de titularidade das patentes foi da Derwent Innovation Index, justamente, porque não se sabe o tamanho e as características do universo de análise. Se a unidade de análise fosse o conjunto de instituições de pesquisa no país, seria difícil obter dados consolidados, uma vez que são pouquíssimas as instituições de pesquisa ou universidades que dispõem de um levantamento exaustivo sobre o número e as características de inovação no Brasil, por isso, optou-se por investigar as patentes de uma base de dados ampla, como é o caso da Derwent. Nesse sentido, obtendo informações de instituições, ano, IPCs, titularidade, entre outros.

\subsection{Coleta e análise dos dados}

Diversos trabalhos são baseados em informações contidas nos documentos de patentes para investigar fenômenos relacionados com a economia da inovação (Krafft et al, 2009; Ventura et al, 2013; Epicoco, 2013; Dal Poz, Silveira, Masago, 2013; Souza, 2013; Bueno, 2016). A amostra de patentes para a investigação das redes tecnológicas foi extraída a partir da base de Derwent Innovations Index durante o período de janeiro de 1976 e de junho de 2016. A base de dados é de propriedade da Thomson Reuters e reúne informações de escritórios e diversos países, incluindo o norte-americano (USPTO), europeu (EPO) e japonês (JPO). A opção por esta base foi devida ao fato de que ela cobre muitos escritórios de patentes. Para analisar as patentes da Petrobras na base de dados da Derwent foi utilizada o programa TI (VantagePoint). Este procedimento é chamado de data mining, cujo princípio maior é tratar grandes quantidades de dados. Porém, esse método não pode ser confundido com a extração de dados, mas sim, a extração de padrões de conhecimentos (coleta, extração, armazenagem, análise e estatísticas) (WU, 2014).

O indicador utilizado para investigar as áreas de especialização tecnológica é a Classificação Internacional de Patentes (IPC), estabelecido pelo Acordo de Estrasburgo 1971, que prevê um sistema hierárquico de símbolos independentes da língua para a classificação de patentes e modelos de utilidade, de acordo com as diferentes áreas de tecnologia a que pertençam. Para investigar a titularidade de 
universidades com a Petrobras é o campo Assignee, que corresponde à titularidade da patente. Somente foi necessário o indexador <Petroleo Brasileiro S.A> não sendo necessário a utilização de outros indexadores, uma vez que o indexador permite recuperar todas as patentes que pertencem a Petrobras, inclusive as depositadas em co-autoria com outras instituições.

A amostra resultou num conjunto de 1.469 patentes da Petrobras. Os dados foram estruturados em padrões (ver Quadro 1). Entre os indicadores estão o ano de depósito, área de especialização, titularidade, entre outros. Com os filtros sobre o nível dos grupos anteriormente definidos pela análise data mining, passou-se para o segundo passo do percurso metodológico: a construção das redes de cooperação tecnológica. O programa VantagePoint relaciona os links de relação entre a Petrobras e outras instituições. Após o tratamento das informações, foram identificados 271 Assignees, ou seja, 271 nomes de titularidade nas patentes. Nessa etapa foram excluídos os nomes de pessoas e outras instituições privadas, nesse estudo são analisadas somente as patentes de titularidade Petrobras x universidades. Dez universidades foram identificadas na rede de cooperação tecnológica. É importante ressaltar que só foram agrupadas as patentes de titularidade Petrobras x universidades, nesse caso, se a titularidade estivesse em nome de pessoa física, como professores, esta não teria sido inserida, exceto nomes de instituições, como por exemplo, Universidade de São Paulo, Universidade Estadual de Campinas.

\section{PROPRIEDADE INTELECTUAL NA PETROBRAS.}

Definir a Petrobras não é tarefa fácil. Isso depende de um amplo estudo que caracterize sua história e trajetória de desenvolvimento, o que envolve uma ampla gama de questões políticas, sociais e econômicas. $\mathrm{O}$ objetivo nesta seção é modesto, na medida em que é uma tentativa (uma vez que se trata de relatar a partir de fontes primárias) o funcionamento de regime de propriedade intelectual da empresa. Por outro lado, é audacioso, uma vez que aponta implicações dessas regulamentações para a formação de redes tecnológicas com as universidades. Tal discussão, na verdade, não diz respeito apenas às atividades relacionadas ao regime de patenteamento da empresa, a tentativa foi de explorar as atividades políticas e econômicas que envolve uma patente.

Primeiramente, é preciso salientar que as atividades de propriedade intelectual (em especial, o patenteamento) estão relacionadas as atividades de Pesquisa e Desenvolvimento (P\&D) de uma empresa. No que tange o setor de petróleo, campo de atividade da Petrobras, Santos (2003) conceitua P\&D como uma atividade de pesquisa e desenvolvimento de interesse público identificados a partir da Lei do Petróleo, cujos benefícios advindos desse tipo de atividade são importantes para o bem-estar social e econômico. Vale mencionar a quantidade de patentes detidas pela empresa. Isso significa que parte da P\&D realizada pela Petrobras, além de gerar benefícios socioeconômicos ao país, também apresentam repercussão positiva para a empresa (Fuck et al, 2007). Uma dessas atividades ligadas ao P\&D da empresa é a seleção de inovações.

A seleção de inovações da Petrobras é definida pela estratégia corporativa (sistema top-dow). O processo funciona da seguinte maneira: após decisão da estratégia corporativa, a tecnologia selecionada passa por um comitê operacional, que fica responsável por ranquear essas tecnologias. Portanto, a estratégia corporativa da empresa é responsável pelo investimento, ou seja, direcionar quais as tecnologias que serão estudadas. As pesquisas são realizadas pelo CENPES, que segue as diretrizes do Plano de Negócios da companhia ${ }^{1}$. Deste modo, são formadas dentro do CENPES as redes temáticas de pesquisa e os núcleos regionais nas áreas de produção, abastecimento, gás natural, energia, desenvolvimento sustentável e gestão tecnológica.

\footnotetext{
${ }^{1}$ Relatório disponível em www.petrobras.com.br O Plano de Negócios e Gestão (2017-2021) prevê investimentos da ordem de US\$ 74,1 bilhões, sendo 82\% deste valor para a área de Exploração e Produção. Nas demais áreas de negócios, os investimentos destinam-se, basicamente, a manutenção das operações e a projetos relacionados ao escoamento da produção de petróleo e gás natural, o que reflete a distribuição dos recursos financeiros totais da Petrobras por área de negócios.
} 
As tecnologias desenvolvidas por esses núcleos de pesquisa são encaminhas para a Gestão de Propriedade Intelectual, através de uma notificação de invenção. Começa-se o processo de regulamentação das tecnologias desenvolvidas. Primeiro é verificado a viabilidade de patenteamento. A análise de viabilidade, normalmente, começa pela análise de patentes publicadas pelo Instituto Nacional de Propriedade Industrial (INPI) e outras fontes de informação tecnológica. O objetivo é saber se a invenção já existe.

No caso de viabilidade de patenteamento da invenção, um documento é gerado para o GT (Gestão de Tecnologia) de licenciamento. Esse GT é formado por um grupo de diretores de várias áreas da Petrobras que se reúne e avalia a tecnologia. A proposta do documento envolve três opções: 1) patentear, 2) publicar $^{2}$ e 3) sigilo. Cabe a essa gerencia decidir o destino da tecnologia inventada. No caso de inovações incrementais, como pequenas mudanças de processo, normalmente, essas não são patenteáveis, para não correr o risco de ser copiadas, então a decisão é manter em sigilo. Cabe também ao GT de licenciamento a responsabilidade de decidir em que país será depositada a patente (cada patente gera uma discussão, uma reunião). Levam-se em consideração os países que a Petrobras tem investimentos ou onde a tecnologia tem possibilidade de negócios. Normalmente, o GT de licenciamento acrescenta países ou retira países (sugerido pelo documento). Nas palavras da Gerência: "Não adianta proteger aquela tecnologia naquele país que a gente sabe que a Petrobras não vai ter nem a médio nem a longo prazo retorno do investimento". Esse procedimento é necessário porque depositar uma patente envolve um alto custo financeiro. Quando a patente é depositada no Brasil o custo financeiro é baixo, porém, a resposta é muito demorada. Já a patente depositada no exterior tem um custo alto, em contrapartida, a resposta tecnológica é rápida.

Um dos problemas apontadas pelo GT de propriedade intelectual da Petrobras refere-se ao depósito de patentes brasileiras no exterior. Quando a tecnologia brasileira é muito interessante, ou seja, rompe paradigmas, esse depósito é dificultado ao máximo. As exigências do escritório são tão emblemáticas, que a Petrobras é forçada a se utilizar de mecanismos jurídicos legais para conseguir depositar a patente no exterior. Esse foi o caso da patente $\mathrm{n}^{\mathrm{o}} 8.232 .082^{3}$ depositada em 2008 e concedida em 2012 pelo escritório americano da USPTO (United States Patent and Trademark Office). A invenção refere-se a um processo para a obtenção de etanol combustível utilizando materiais de resíduos agrícolas e compostos agroindustriais de lignocelulose, especialmente, o bagaço de cana de açúcar. A planta piloto desta tecnologia foi instalada em 2007 no Brasil (Rio de Janeiro), sendo instalada a planta piloto nos Estados Unidos (Upton, Wyoming) em parceria com a Blue Sugars no ano de 2011. Segundo a Gerência de Propriedade Intelectual, nessa área, que utiliza microrganismos para obtenção de biocombustíveis, o depósito de patentes vem acontecendo de minuto a minuto.

No que se refere ao tempo de concessão de uma patente no Brasil ${ }^{4}$, o que a Petrobras tem feito para resolver esse problema é depositar no Brasil e logo em seguida nos outros países. Como no exterior a concessão sai antes, a estratégia é que a partir do momento do depósito, como existe uma expectativa da empresa de que aquela tecnologia seja concedida (uma vez que já que foi feito um estudo de viabilidade), a Petrobras libera o uso e não fica aguardando a concessão. Um fator que deve ser considerado é que a política da Petrobras sempre foi de proteger a tecnologia para ter liberdade de usar, não para receber retornos sobre licenciamentos, vendas, entre outros. Entretanto, recentemente, isso mudou. A nova

\footnotetext{
${ }^{2} \mathrm{O}$ motivo mais frequente da publicação é a divulgação da tecnologia por universidades ou instituições que estão trabalhando no mesmo assunto, e se de fato não for de interesse da Petrobras, neste caso, simplesmente divulga-se para contribuição científica.

${ }^{3}$ Link de acesso para a patente http://patft.uspto.gov/netacgi/nphParser?Sect1=PTO2\&Sect2=HITOFF\&u=\%2Fnetahtml\%2FPTO $\% 2$ Fsearch-

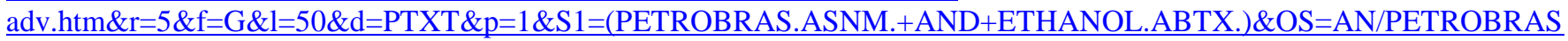
$+\mathrm{AND}+\mathrm{ABST} / \mathrm{ETHANOL \& RS=(AN/PETROBRAS+AND+ABST/ETHANOL)}$ Essa patente, além dos Estados Unidos, foi concedida em diversos outros países, entre eles, a Colômbia. Este país, recentemente, tem investido em pesquisas de biocombustíveis.

${ }^{4}$ A área de química demora em média dez anos para receber a concessão da patente e a área de mecânica, em média oito anos, enquanto no exterior, espera-se em média de dois a cinco anos.
} 
política é de patentear tudo que pode ter retorno de investimento, mesmo que não seja especificamente para o setor do petróleo. Isso ocorreu após a quebra do monopólio, e a Petrobras passou a se interessar em comercializar suas tecnologias. A contrapartida é que essa nova política teve implicações importantes, em especial, no âmbito das universidades. Ponto tratado a seguir.

\section{POLÍTICAS E IMPLICAÇÕES: O IMPACTO NAS UNIVERSIDADES}

O cenário de patenteamento da companhia, de acordo com a Gerencia de Propriedade Intelectual, foi a criação, em 2001, do prêmio inventor ${ }^{5}$, que começou a triplicar o número de notificações de invenção. O prêmio é fixado em um valor de um nível de salário, que vai depender do grau de importância da patente para a Petrobras. Se for uma tecnologia que não tem muita importância, atribui-se uma nota, se aquilo for importante e pode ser aplicado em várias áreas da Petrobras, outra nota será atribuída. Os valores são atribuídos de acordo com essa nota de importância. Então, quanto maior a nota, maior será o prêmio, e este prêmio é dividido entre os inventores da tecnologia. Se somarmos esse instrumento de apoio à inovação feito pela Petrobras à combinação de um conjunto de políticas de incentivo de inovação, em geral, ocorridos ao final das décadas de 1990 e meados de $2000^{6}$, ao passo que em 2006, a Agencia Nacional de Petróleo (ANP) ter regulamentado uma cláusula de obrigação contratual para companhias petrolíferas atuantes no Brasil destinarem pelo menos $1 \%$ do faturamento bruto para investimentos em Pesquisa e Desenvolvimento (a partir da Lei de Inovação Federal - Lei 10.973/04), obtemos um novo cenário de regulação e mudanças não somente no âmbito da Petrobras, mas também de uma infraestrutura científica e tecnológica no país. Isso porque, depois da lei da inovação, a Petrobrás começou a fazer muitos trabalhos com universidades nacionais e internacionais, e instituições de pesquisa. A lei determina que a Petrobras é obrigada a investir $1 \%$ do faturamento em pesquisa (ela optou por investir $0,5 \%$ internamente e $0,5 \%$ externamente), a partir disso a empresa começou a fazer muitos convênios com universidades, além dos institutos de pesquisa, forçando as redes tecnológicas nacionais e internacionais de pesquisa.

A iniciativa foi criada em 1998 e a verba começou a ser aplicada somente em 2005. Primeiro surgiram os CTPETROS (CNPq lançou os editais), posteriormente, a Petrobras ficou responsável em aplicar esse dinheiro. O problema é que a verba ficou represada por muitos anos, e a Petrobras corria o risco de pagar uma multa altíssima por não aplicar a lei da inovação. O processo complicou-se, porque o valor ficou estagnado por um tempo, uma vez que a Petrobras não sabia como selecionar os agentes que receberiam esses recursos. Uma solução da Petrobras para resolver de imediato esse problema foi destinar a aplicação dessa verba em infraestrutura de pesquisa. Ocorreu um boom para comprar equipamentos, construir prédios, criar laboratórios de pesquisa etc., esse fato pode explicar o rápido crescimento da infraestrutura cientifica e tecnológica que ocorreu no país nos anos 2000. O relatório de um estudo preliminar do Instituto de Pesquisa Econômica Aplicada (IPEA), lançado em 2014, destacou o salto brasileiro em infraestrutura de pesquisa no país (DE NEGRI et al, 2014).

Tabela 1. Infraestruturas mapeadas, segundo ano de operação

\begin{tabular}{l|l|l}
\hline Período & $N^{o}$ de infraestruturas & $\%$ \\
\hline $1970-1979$ & 110 & $6,3 \%$ \\
\hline $1980-1989$ & 193 & $11,0 \%$ \\
\hline $1990-1999$ & 410 & $23,3 \%$ \\
\hline $2000-2009$ & 654 & $37,2 \%$ \\
\hline
\end{tabular}

Fonte: Adaptado De Negri (IPEA, 2014).

\footnotetext{
${ }^{5}$ Informações sobre o prêmio pode ser acessada através do site: http://sites.petrobras.com.br/minisite/premiotecnologia/apresentacao/apresentacao.asp

${ }^{6}$ O período inicia o marco legal para inovação no Brasil, derivadas da Lei de Inovação Federal (Lei 10.973/04).
} 
Segundo dados do relatório do IPEA, mais de 70\% das instituições se modernizaram nos anos de 2008 a 2012. Entretanto, a Petrobras não aplicou mais a verba para destinação de infraestrutura, somente para pesquisas científicas e tecnológicas. Isto ocorreu porque a Petrobras sofreu uma mudança importante. Foi necessário criar uma nova Gerencia de Relacionamento $\left(\mathrm{RCT}^{7}\right)$ para gerenciar esses recursos. Nas palavras da Gerencia: "A Petrobras nunca teve esse tipo de relacionamento (quem fazia era o CNPq), então, precisou criar uma gerencia, porque a Petrobras não sabia nem como fazer prestação de contas disso, como iria aportar essa verba, que era enorme".

Além desses incentivos que aportaram o salto de patenteamento da empresa, outra mudança significativa refere-se a mudança após a quebra do monopólio. A Petrobras começou a negociar suas tecnologias. Isto porque, um percentual do que é patenteado passou a ser licenciado, e os inventores também ganharam com isso. Significa que se a empresa está ganhando royalties com a tecnologia, os inventores também recebem uma porcentagem desses lucros. Em contrapartida, muitos inventores começaram a participar de uma mesma patente (de um ou dois inventores, as notificações começaram a apresentar mais de 20 inventores em uma mesma patente). A hipótese é que além do prêmio inventor existe um incentivo pessoal de trabalho em colaboração.

$\mathrm{O}$ aumento de inventores em uma mesma patente implicou desafios para a Empresa. Ocorreu que as patentes depositadas fora do país, em especial, no escritório americano da USPTO, o número alto de inventores começou a dificultar as concessões de patentes. De acordo com o GT de Propriedade Intelectual, nos Estados Unidos, quando chega uma patente com vários inventores, uma das exigências é informar qual foi a contribuição de cada inventor a partir do que está sendo reivindicado como invenção. O Setor de Propriedade Intelectual normalmente consegue realizar a exigência, porém, sempre com muita dificuldade, o que acaba atrasando o processo de patenteamento.

No entanto, se de um lado, o cenário de aumento expressivo de inovações no âmbito da Petrobras implicou em mudanças organizacionais internas, essas também implicaram em mudanças nas universidades. Até então (antes dos anos 2000) não existia uma cadeira eletiva para fazer o trabalho de informação tecnológica nas universidades junto a Petrobras, mas, diante dos novos relacionamentos entre a Petrobras e as universidades (devido a obrigatoriedade da lei) isso começou a mudar. A primeira ação concreta: o contrato. Todo contrato de cooperação passou a ter uma cláusula de propriedade intelectual, que diz que se daquele contrato surgir um fruto que pode ser patenteável, qual será a parcela da Petrobras e qual será a parcela da universidade. Antes, tudo que era desenvolvido com universidades, a titularidade era inteiramente da Petrobras (atualmente, nunca mais foi $100 \%$ da Petrobras). Com o acordo que as universidades fizeram através da Associação Nacional dos Dirigentes das Instituições Federais de Ensino Superior (ANDIFES), três opções de titularidade são possíveis: 80\% (Petrobras) e 20\% (Universidades); $50 \%$ e $50 \%$; e, $20 \%$ e $80 \%$. Essa titularidade dependerá do interesse da Petrobras na tecnologia. Quando se trata de um assunto que a Petrobras nunca vai colocar em prática, por exemplo, um microrganismo que vai servir para uso fora da indústria de petróleo apenas 20\% fica para Petrobras e os restantes $80 \%$ para a universidade. A Petrobras nunca cobrou o custo de propriedade intelectual das universidades, mas, recentemente instalou-se esta cobrança por parte da Petrobras, o que forçou as universidades a gerenciar suas inovações ${ }^{8}$. As universidades foram obrigadas a criar os Núcleos de Informação Tecnológica, os NITs, que são responsáveis pelo gerenciamento das patentes e pelo incentivo a inovação 9 .

\footnotetext{
${ }^{7}$ Antes de criar a RCT para controle dos relacionamentos internos e externos da Petrobras, foi levantado pela Gerencia de Propriedade Intelectual um problema relativo aos vários pesquisadores de universidades que figuravam em vários projetos da Petrobras que somavam mais de 44 horas semanais. Para resolver esse problema foi necessário criar a Gerencia para realizar esse controle. Essas regras foram sendo cridas ao longo do tempo, e melhoradas por vias de aprendizado.

${ }^{8} \mathrm{Na}$ prática o que acontece é diferente. Quando a universidade faz um projeto com a Petrobras, normalmente, no contrato há um custo com a patente, e no fim quem acaba pagando os custos dessa patente é a Petrobras.

${ }^{9}$ Outro fator importante é que o CNPq começou a dar mais pontos (incentivos) para patentes do que para artigos, incentivando as universidades ao patenteamento.
} 


\section{4. ÁREAS TECNOLÓGICAS E A REDE DE COOPERAÇÃo}

\subsection{As áreas tecnológicas}

Diversos trabalhos são baseados em informações contidas nos documentos de patentes para investigar fenômenos relacionados com a economia da inovação (Krafft et al, 2009; Ventura et al, 2013; Epicoco, 2013; Dal Poz, Silveira, Masago, 2013; Souza, 2015). Para compor a amostra de patentes relacionadas com o tema 'PETROBRAS' para a investigação de redes tecnológicas foi extraída a partir da base ISI Derwent Innovations Index, durante o período de janeiro de 1976 e de junho de 2016. O campo utilizado para levantar as patentes da Derwent foi: campo: Depositante: Petrobras. O resultado da amostra é de um total de 1.469 patentes.

A partir dessa amostra são gerados os indicadores de análise deste estudo (Quadro 1), este procedimento é chamado de data mining, cujo princípio maior é tratar grandes quantidades de dados. Porém, esse método não pode ser confundido com a extração de dados. E sim é um método de extração de padrões de conhecimentos (coleta, extração, armazenagem, análise e estatísticas) (WU, 2014). O Programa de computador utilizado como instrumento de análise (data mining) é o The VantagePoint.

Quadro 1. Indicadores da amostra de Patentes. Petrobras - Derwent.

\begin{tabular}{|l|l|l|}
\hline Filtro (campo) & \multicolumn{2}{l}{ Número de ítens $\%$ Cobertura dos dados } \\
\hline Patentes & 1,469 & $100 \%$ \\
\hline Basic Patent Year & 43 & $100 \%$ \\
\hline Derwent Accession Number & 1,469 & $100 \%$ \\
\hline International Classifications 8 & 2,098 & $100 \%$ \\
\hline International Classifications 8 (4-digit) & 243 & $100 \%$ \\
\hline Patent Assignees & 345 & $100 \%$ \\
\hline Priority Countries & 28 & $100 \%$ \\
\hline Priority Years & 44 & $100 \%$ \\
\hline Publication Type & 1 & $100 \%$ \\
\hline Title & 1,467 & $100 \%$ \\
\hline US Patent Years & 38 & $25 \%$ \\
\hline
\end{tabular}

Fonte: Dados da pesquisa.

O primeiro indicador analisado é o basic patent year. Este indicador permite saber o ano de depósito da patente. O ano de 1975 não apresenta patente. O período de 1984 e 1993 houve uma variação entre 15 e 40 patentes. Em 1994 atinge-se o pico de 52 patentes. Depois, uma variação de 20 a 40 patentes até 2003. A partir de 2004 o número de patentes varia entre 50 e 70, atingindo o pico máximo de 98 patentes no ano de 2007.

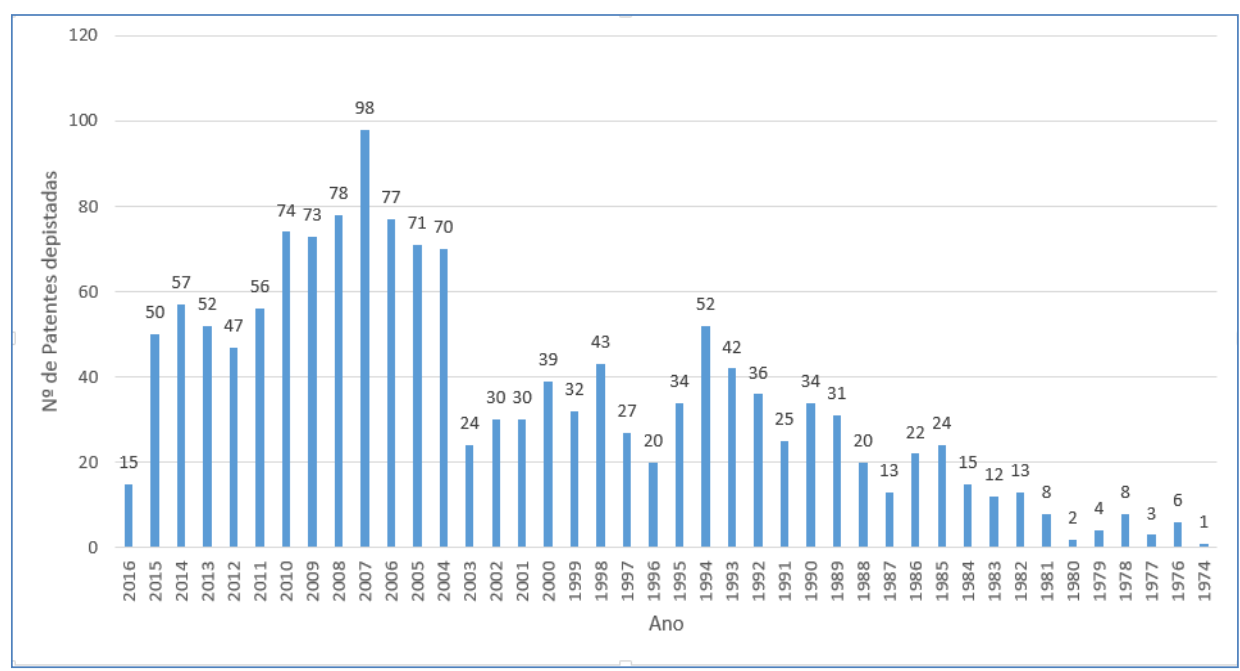

Gráfico 3. Patentes depositadas por ano - Petrobras.

Fonte: Dados da pesquisa. 
Com o filtro International Classifications 8 (4 dígitos), indicador da classificação de IPCs, é possível saber quais são as áreas do conhecimento das patentes. A Classificação Internacional de Patentes (IPC ${ }^{10}$ ), estabelecida pelo Acordo de Estrasburgo, em 1971, prevê um sistema hierárquico de símbolos independentes da língua para a classificação de patentes e modelos de utilidade, de acordo com as diferentes áreas de tecnologia a que pertençam, cujas áreas tecnológicas podem estar classificadas de A a H. Sendo:

SEÇÃO A - NECESSIDADES HUMANAS; SEÇÃO B - OPERAÇÕES DE PROCESSAMENTO; TRANSPORTE; SEÇÃO C-QUÍMICA; METALURGIA; SEÇÃO D - TÊXTEIS; PAPEL; SEÇÃO ECONSTRUÇÕES FIXAS; SEÇÃO F- ENGENHARIA MECÂNICA; ILUMINAÇÃO; AQUECIMENTO; ARMAS; EXPLOSÃO; SEÇÃO G-FÍSICA; SEÇÃO H-ELECTRICIDADE.

No filtro, foram identificadas 243 classes de tecnologias, destas, são mais de 2.098 sub-classes de especialização. Este estudo, em princípio, está limitado a análise das classes de 4 dígitos. O Quadro 2 mostra as principais áreas de especialização tecnológica da amostra de patentes da Petrobras.

Quadro 2. Principais áreas tecnológicas - Petrobras.

\begin{tabular}{|r|r|r|}
\hline Patentes & Frequência & International Classifications 8 (4-digit) \\
\hline $\mathbf{3 8 5}$ & $\mathbf{7 1 2}$ & E21B \\
\hline $\mathbf{1 8 5}$ & $\mathbf{4 5 2}$ & C10G \\
\hline $\mathbf{1 8 4}$ & $\mathbf{5 4 1}$ & B01J \\
\hline $\mathbf{1 0 3}$ & $\mathbf{1 8 9}$ & F16L \\
\hline $\mathbf{9 2}$ & $\mathbf{1 7 2}$ & B63B \\
\hline $\mathbf{9 0}$ & $\mathbf{1 4 6}$ & $\mathbf{G 0 1 N}$ \\
\hline 76 & 182 & C07C \\
\hline 75 & 110 & B01D \\
\hline 46 & 82 & C09K \\
\hline 45 & 93 & C10L \\
\hline 43 & 83 & B08B \\
\hline 38 & 70 & C01B \\
\hline 32 & 57 & C02F \\
\hline 31 & 41 & G01F \\
\hline 29 & 39 & E02B \\
\hline 27 & 59 & C10B \\
\hline 26 & 48 & B65D \\
\hline 26 & 44 & C08L \\
\hline 25 & 90 & C08F \\
\hline 22 & 36 & C12P \\
\hline
\end{tabular}

Fonte: Dados da pesquisa.

A área de maior especialização tecnológica, atingindo 385 patentes com uma frequência de 712 vezes, é a classe E21B. Esta classe pertence a área tecnológica de IPC - "E - relacionada a construções fixas, na categoria - 21 - de perfuração do solo, mineração, e Seção B: perfuração do solo ou rocha; obtenção de óleo, gás, água, materiais solúveis ou fundíveis ou uma lama de minerais de poços”.

Em segundo lugar, está a classe de IPC C12G, atingindo 185 patentes com uma frequência de 452 vezes. Esta classe pertence a área tecnológica de IPC - "C - relacionada a Química; Metalurgia, na categoria 10 - indústrias do petróleo, do gás ou do coque; gases técnicos contendo monóxido de carbono; combustíveis; lubrificantes; turfa, e Seção G: "craqueamento de óleos hidrocarbonetos; produção de misturas hidrocarbonetos líquidos, p. ex. por hidrogenação destrutiva, oligomerização, polimerização;

${ }^{10}$ Disponível no site: http://ipc.inpi.gov.br/ipcpub/\#refresh=page 
recuperação de óleos hidrocarbonetos de óleo de xisto, areia oleaginosa ou gases; refino de misturas principalmente consistindo de hidrocarboneto; reforma de nafta; ceras minerais.

Em terceiro lugar figura a classe B01J, atingindo 184 patentes com uma frequência de 541 vezes (ultrapassando a frequência da classe $\mathrm{C} 12 \mathrm{G}$ ). Esta classe pertence à área tecnológica de IPC - "B operações de processamento; transporte, categoria - 01 - processos ou aparelhos físicos ou químicos em geral, e Seção J: processos químicos ou físicos, p. ex. catálise, química coloidal; aparelhos pertinentes aos mesmos.

Em quarto lugar figura a classe F16L, atingindo 103 patentes com uma frequência de 189 vezes. Esta classe pertence à área tecnológica de IPC - F - engenharia mecânica; iluminação; aquecimento; armas; explosão, categoria - 16 - elementos ou unidades de engenharia; medidas gerais para assegurar e manter o funcionamento efetivo de máquinas ou instalações; isolamento térmico em geral, e Seção L: tubos; juntas ou acessórios para tubos; suportes para tubos, cabos ou tubulação de proteção; meios para isolamento térmico em geral.

Com 92 patentes e uma frequência de 172 vezes em quinto lugar figura a classe de IPC B63B. Esta classe pertence à área tecnológica de IPC - B - operações de processamento; transporte, categoria - 63 - navios ou outras embarcações; equipamento correlato, e Seção B: navios ou outras embarcações; equipamento para a navegação (disposições para ventilação, aquecimento, resfriamento ou condicionamento de ar em embarcações; Infraestruturas flutuantes como elementos de apoio de dragas ou de máquinas de terraplenagem).

Em sexto lugar tem-se a classe G01N, atingindo a quantia de 90 patentes com 146 frequências. Esta classe pertence à área tecnológica de IPC - G - Física, categoria - 01 - medição; teste, e Seção N: investigação ou análise dos materiais pela determinação de suas propriedades químicas ou físicas, materiais" inclui meios sólidos, líquidos, e gasosos, p. ex. a atmosfera.

A Classe C12P ocupa o vigésimo lugar e corresponde a área de especialização de processos de fermentação ou processos que utilizem enzimas para sintetizar uma composição ou composto químico desejado ou para separar isômeros ópticos de uma mistura racêmica. Principalmente, esta classe se refere a processos utilizados para produção de biocombustíveis nas patentes da estatal.

As áreas de especialização da Classe C - Química e Metalurgia - é a principal área de tecnologia das patentes da Petrobras (somando 10 áreas de especialização), em seguida (com 5 áreas) temos a classe B - operações de processamento; transporte. Depois (com 2 áreas) estão as classes E e G. A classe E construções fixas, e a classe $\mathrm{g}$ - física. Com 1 área temos a classe $\mathrm{f}$ —engenharia mecânica; iluminação; aquecimento; armas; explosão.

Para avaliar a correlação das áreas tecnológicas da amostra de patentes, foi aplicada a matriz de adjacência a partir do programa VantagePoint. O algoritmo do programa TI aplica a rede para um esquema interativo orientado por uma árvore de extensão mínima da rede, a fim de gerar coordenadas para os nós expressando a correlação.

Inserimos as principais classes de tecnologias e verificamos os grafos de correlação em rede. Se existe relação, esta deve apresentar os níveis de correlação, positiva ou negativa, entre as áreas tecnológicas.

A Figura 1 mostra o resultado. Foi necessário quebrar a rede em partes para entender as correlações. $\mathrm{O}$ bloco de tecnologias E21B está relacionado positivamente com as classes F16L, E02B, B63B, B08B, C09K, G01N, C02F, G01F, B01D, B65D e C01B e negativamente com as classes B01J, C08L, C07C, C10L, C10B, C08F. 
Isto quer dizer que a classe E21B que corresponde a construções fixas em perfuração de rochas, solos e mar, está relacionada negativamente com a classe $\mathrm{C} 12 \mathrm{P}$ que corresponde a estudos de bioquímica e biocombustíveis e positivamente com a classe F16L que corresponde a tecnologias de engenharia mecânica; iluminação; aquecimento; armas e explosão. $O$ resultado mostra que as patentes estão separadas em dois blocos tecnológicos. Um de tecnologias para produção de química verde e bioenergia, como produtos de biorrefinarias, e o outro bloco de tecnologias específicas para a exploração e produção de petróleo, especialmente para estruturas (como off-Shore) e engenharia.

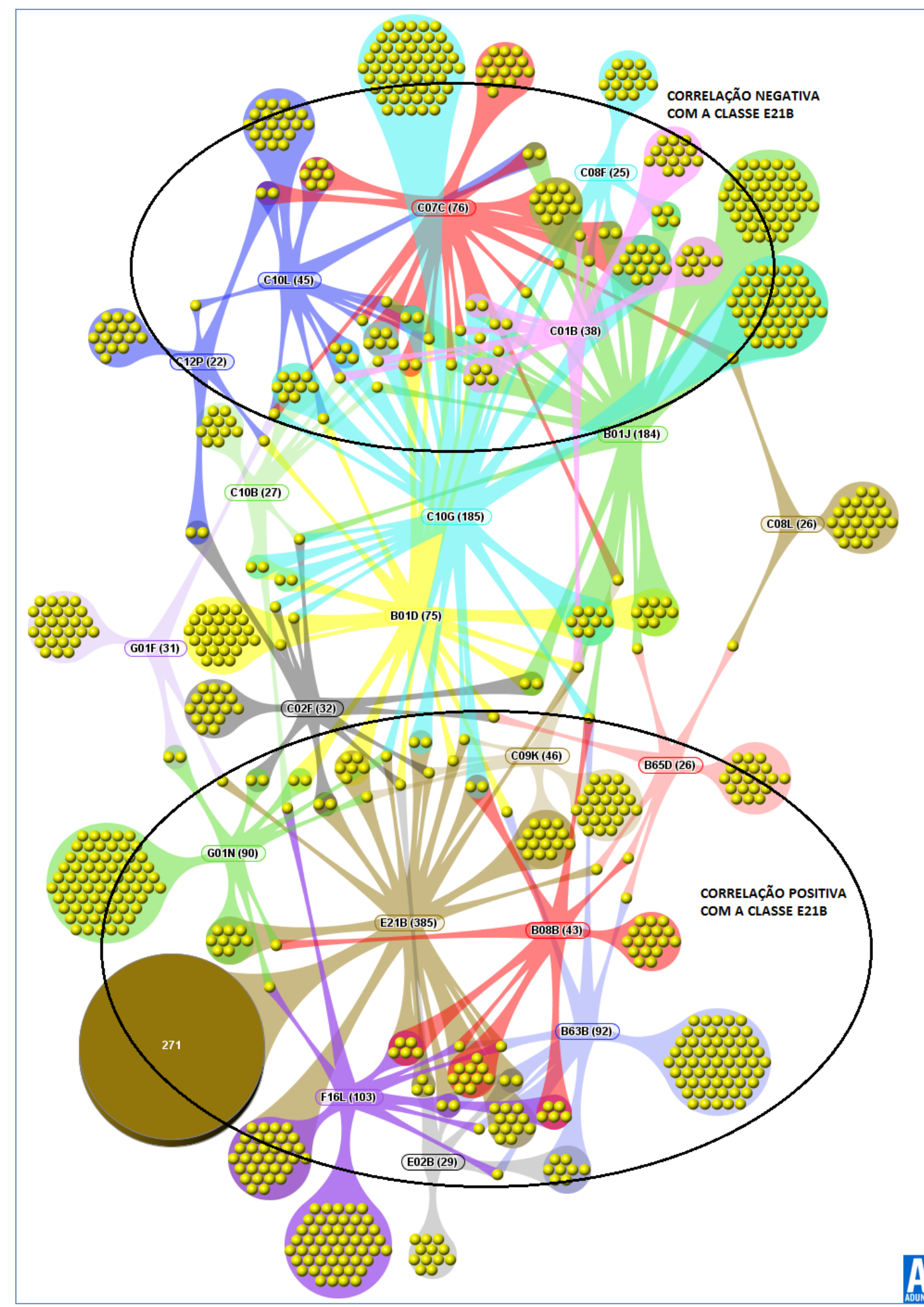

Figura 1. Rede de IPCs nas áreas de especialização da amostra de patentes. Fonte: Dados da pesquisa. 


\subsection{As redes de cooperação tecnológica Petrobras e universidades}

As redes tecnológicas são investigadas a partir da coautoria nas patentes das áreas tecnológicas que foram mostradas anteriormente. O objetivo é identificar as instituições em parceria tecnológica com a Petrobras. A colaboração científica é definida como a interação ocorrendo dentro de um contexto social entre dois ou mais cientistas, (redes) que objetivam a partilha de significado e realização de tarefas em relação a uma solução mutuamente compartilhada (Leydesdorff et al., 2008). As redes são representadas por grafos, que ilustram a rede representada da seguinte maneira: um grafo direcionado consiste de um conjunto $\mathrm{V}$ de vértices (ou nós), um conjunto $\mathrm{E}$ de arestas (links ou Edges), $\mathrm{E} \rightarrow \mathrm{V}$, onde (e) é a fonte $\mathrm{e}$ (v) é o alvo da aresta direcionada (Goyal, 2007). Então, qualquer conjunto de elementos no qual alguns deles estão conectados em pares através de links será representado. Na rede de coautoria os vértices são pesquisadores e as arestas indicam algum tipo de colaboração, como a titularidade em conjunto. Neste caso, dois vértices estão relacionados se dois pesquisadores são coautores de, ao menos, uma mesma patente.

Esse método foi aplicado a partir do Programa VantagePoint para identificar as relações de titularidade nas patentes. Observe a Figura 2. Esta rede mostra as relações de coautoria com a Petrobras. Note a coautoria da Universidade Federal do Rio de Janeiro (em vermelho no cluster), são 20 patentes, destas, dezesseis são em titularidade com a Petrobras, uma em titularidade com a Universidade de Valencia e três em titularidade com a Pontifícia Universidade Católica do Rio. Todas formam um cluster de cooperação tecnológica entre si e a Petrobras. Ao contrário das outras universidades, em que a relação é somente delas com a Petrobras. Como mostra a rede da Universidade Estadual de Campinas (verde no cluster), são cinco patentes em titularidade com a Petrobras, e a relação não envolve outras instituições.

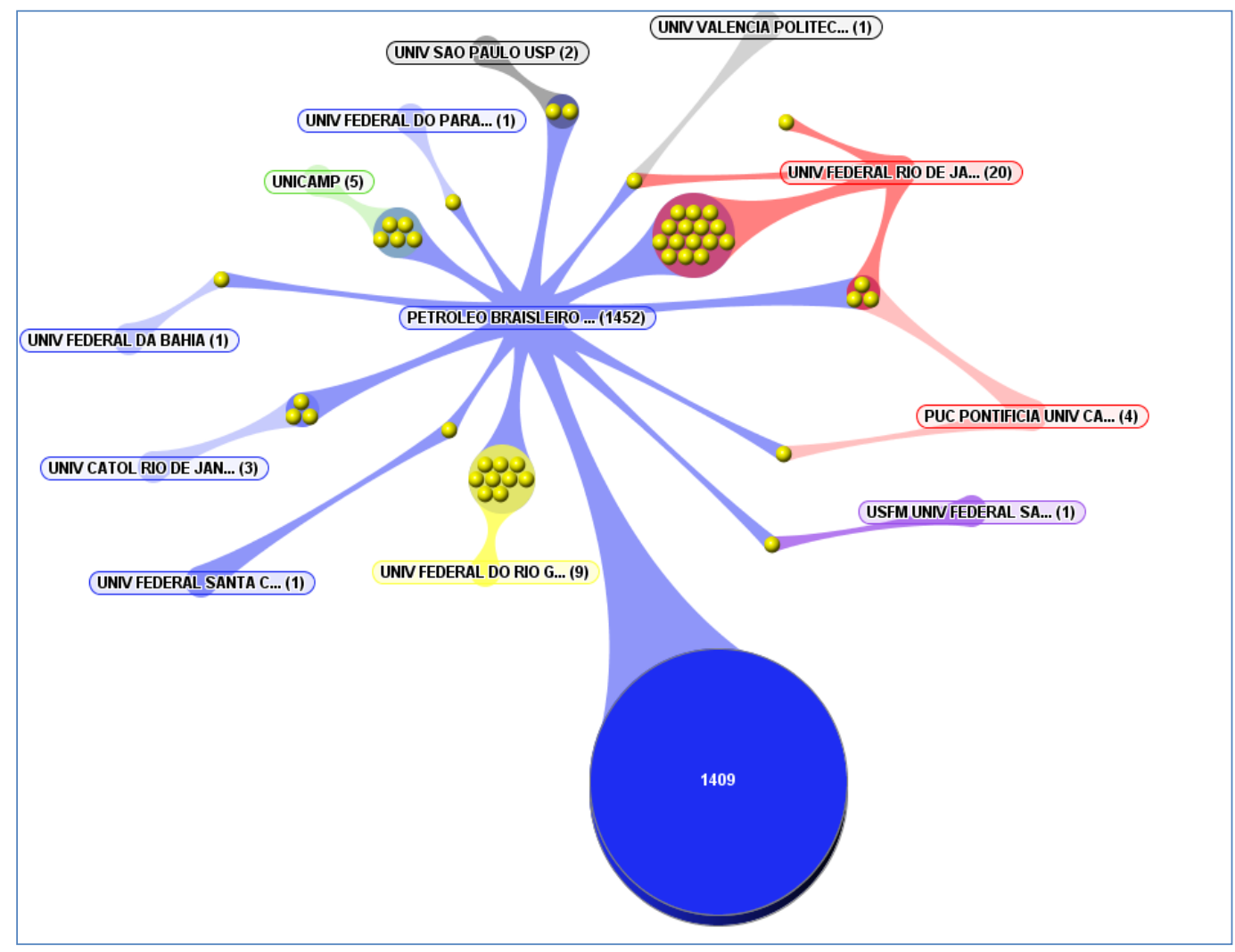

Figura 2. Rede tecnológica - Titularidade nas patentes - Petrobras x Universidades. Fonte: Dados da pesquisa.

Depois da UFRJ o destaque é a Universidade Federal do Rio Grande do Sul com nove patentes. Em seguida está a Pontifícia Universidade Católica do Rio de Janeiro com 4 patentes com a Petrobras e UFRJ, e outras três com a Petrobras. A Universidade de São Paulo tem duas patentes. Depois temos a Universidade de Santa Catarina, Universidade Federal da Bahia, Universidade Federal do Pará, 
Universidade de Valencia, Universidade Federal de Santa Maria, todas com uma patente cada. As áreas de especialização da rede tecnológica da Petrobras com as universidades mostradas são mostradas nas tabelas a seguir. A Tabela 2 mostra as classes de IPCs das patentes de titularidade Petrobras e Universidade Federal do Rio de Janeiro (UFRJ).

\begin{tabular}{|c|c|}
\hline \multicolumn{2}{|r|}{ Tabela 2 - IPC das Patentes (4-digit) Petrobras e UFRJ. } \\
\hline № de patentes & Descrição \\
\hline 4 & $\begin{array}{l}\text { - C10L: combustíveis não incluídos em outro local; gás natural; gás natural sintético; gás liquefeito de } \\
\text { petróleo; uso de aditivos em combustíveis ou ao fogo; acendedores de fogo. }\end{array}$ \\
\hline 3 & $\begin{array}{l}\text { - C12P: processos de fermentação ou processos que utilizem enzimas para sintetizar uma } \\
\text { composição ou composto químico desejado ou para separar isômeros ópticos de uma mistura } \\
\text { racêmica. }\end{array}$ \\
\hline 2 & $\begin{array}{l}\text { - } \quad \text { B01D: separação de sólidos de outros sólidos por via úmida, por meio de peneiras ou mesas } \\
\text { pneumáticas, por outros métodos a seco; separação magnética ou eletrostática de materiais sólidos } \\
\text { dos materiais sólidos ou de fluidos. } \\
\text { - } \quad \text { B01L: processos químicos ou físicos, p. ex. catálise, química coloidal; aparelhos pertinentes aos } \\
\text { - } \quad \text { C07C: compostos acíclicos ou carbocíclicos (preparação de compostos macromoleculares). } \\
\text { - } \quad \text { C10G: craqueamento de óleos hidrocarbonetos; produção de misturas hidrocarbonetos líquidos } \\
\text { - C10M: composições lubrificantes (composições para perfuração de poços). } \\
\text { - F16L: tubos; juntas ou acessórios para tubos; suportes para tubos, cabos ou tubulação de } \\
\text { proteção; meios para isolamento térmico em geral. }\end{array}$ \\
\hline 1 & 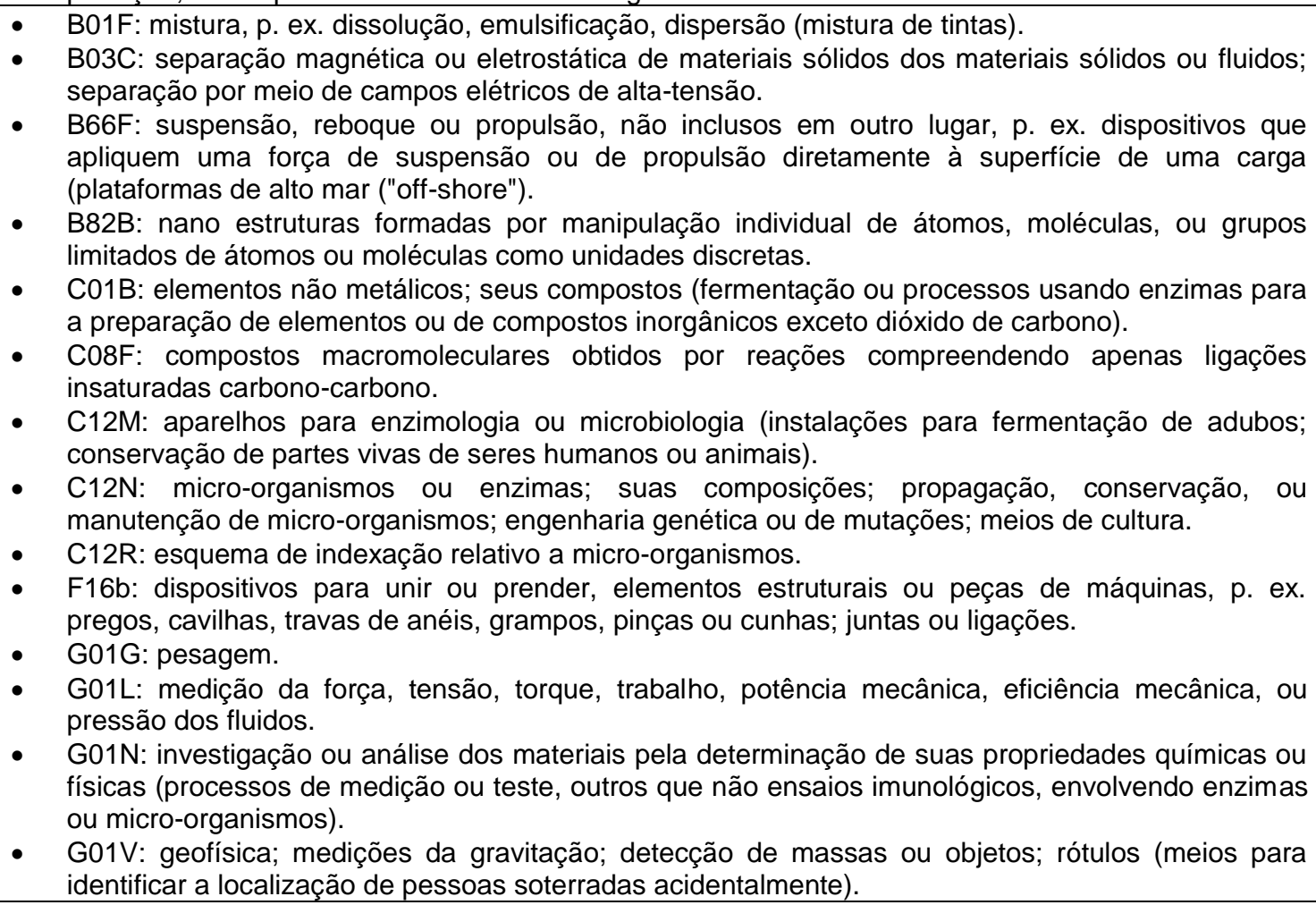 \\
\hline
\end{tabular}

Fonte: Dados da pesquisa. 
Tabela 3 - IPC das Patentes (4-digit) Petrobras e UFRGS.

\begin{tabular}{|c|c|}
\hline \multicolumn{2}{|r|}{ Tabela 3 - IPC das Patentes (4-digit) Petrobras e UFRGS. } \\
\hline № de patentes & Descrição \\
\hline 4 & $\begin{array}{l}\text { - C09K: materiais para aplicações diversas, não incluídas em outro local; aplicaçôes de materiais não } \\
\text { incluídos em outro local. }\end{array}$ \\
\hline 2 & $\begin{array}{l}\text { - C07C: compostos acíclicos ou carbocíclicos (preparação de compostos macromoleculares; } \\
\text { produção de compostos orgânicos por eletrólise ou eletroforese). } \\
\text { - C07F: compostos acíclicos, carbocíclicos ou heterocíclicos contendo outros elementos que não o } \\
\text { carbono, o hidrogênio, o halogênio, o nitrogênio, o enxofre, o selênio ou o telúrio. }\end{array}$ \\
\hline 1 & 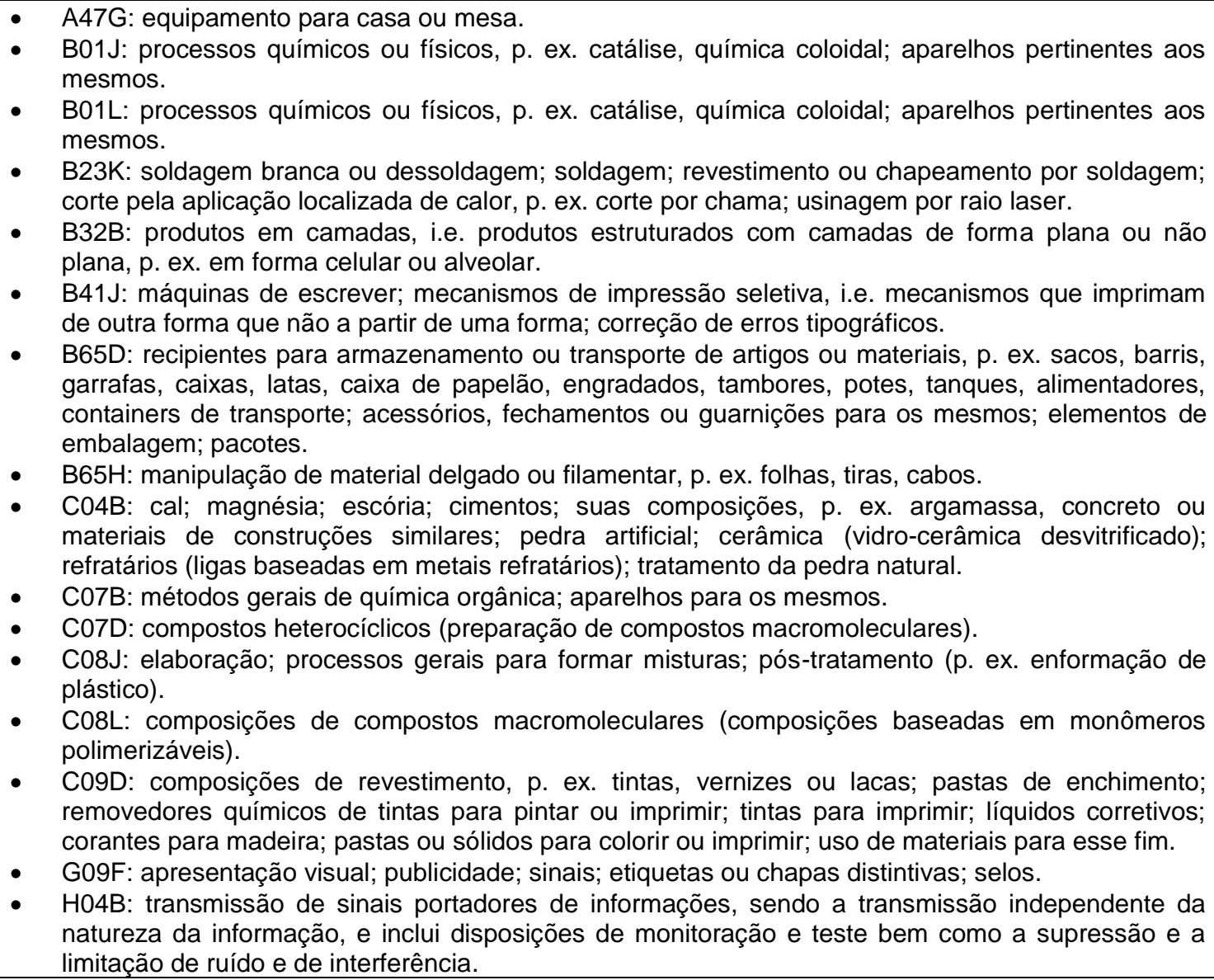 \\
\hline
\end{tabular}

Fonte: Dados da pesquisa.

No caso da rede entre Petrobras e UFGRS as áreas tecnológicas são apresentadas na Tabela 3. Diferentemente da UFRJ, esta rede apresenta o desenvolvimento de novos produtos a partir dos materiais, principalmente para uso doméstico, como utensílios.

Já a rede tecnológica da Universidade Estadual de Campinas e a Petrobras, as classes são apresentadas na Tabela 4. As áreas envolvem procedimentos de exploração de novos materiais, inovações incrementais, nas áreas de química, metalurgia, engenharias e explosão. 
Tabela 4 - IPC das Patentes (4-digit) Petrobras e UNICAMP

\begin{tabular}{|c|c|}
\hline № de patentes & Descrição \\
\hline 2 & $\begin{array}{l}\text { - C10J: produção de gases contendo monóxido de carbono e hidrogênio a partir de matérias } \\
\text { carbonáceas sólidas por processos de oxidação parcial envolvendo oxigênio ou vapor (gaseificação } \\
\text { subterrânea de minerais e; carburação do ar ou de outros gases). }\end{array}$ \\
\hline 1 & $\begin{array}{l}\text { - } \quad \text { B01J: processos químicos ou físicos, p. ex. catálise, química coloidal; aparelhos pertinentes aos } \\
\text { mesmos (processos ou aparelhos para usos específicos). } \\
\text { - C09K: materiais para aplicações diversas, não incluídas em outro local; aplicaçôes de materiais não } \\
\text { incluídos em outro local. } \\
\text { - C10B: destilação destrutiva de substâncias carbonáceas para produção de gás, coque, alcatrão ou } \\
\text { - } \quad \text { c10Gstâncias similares (craqueamento de óleos; gaseificação subterrânea de minerais). } \\
\text { ex. por hidrogenação destrutiva, oligomerização, polimerização (craqueamento de hidrogênio ou } \\
\text { gás de síntese; craqueamento ou pirólise de hidrocarboneto em hidrocarbonetos individuais ou } \\
\text { suas misturas de constituição definida ou especificada; craqueamento em coque; recuperação de } \\
\text { óleos hidrocarbonetos de óleo de xisto, areia oleaginosa ou gases; refino de misturas } \\
\text { principalmente consistindo de hidrocarboneto; reforma de nafta; ceras minerais). } \\
\text { F28D: condensadores de vapor de água ou de outros vapores (condensação de vapores; } \\
\text { condensação durante o pré-tratamento de gases antes da precipitação eletrostática de partículas } \\
\text { dispersas; instalações de motores a vapor tendo condensadores; liquefação de gases; detalhes das } \\
\text { disposições de troca de calor e de transferência de calor de aplicação geral). }\end{array}$ \\
\hline
\end{tabular}

Fonte: Dados da pesquisa.

A rede de cooperação tecnológica entre a USP e a Petrobras, as áreas compreendem as tecnologias para tratamento de águas residuais e da química verde (por exemplo, óleos para cosméticos e novos materiais para uso em produtos, como tintas).

\begin{tabular}{|c|c|}
\hline \multicolumn{2}{|r|}{ Tabela 5 - IPC das Patentes (4-digit) Petrobras e USP } \\
\hline № de patentes & Descrição \\
\hline 1 & $\begin{array}{l}\text { - } \quad \text { B01D: separação de sólidos de outros sólidos por via úmida, por meio de peneiras ou mesas } \\
\text { pneumáticas, por outros métodos a seco; separação magnética ou eletrostática de materiais sólidos } \\
\text { dos materiais sólidos ou de fluidos, separação por meio de campos elétricos de alta-tensão; } \\
\text { centrífugas; aparelhos de vórtice; prensasper separa espremer o líquido de materiais que o } \\
\text { contenham } \\
\text { B01J: processos químicos ou físicos, p. ex. catálise, química coloidal; aparelhos pertinentes aos } \\
\text { mesmos (processos ou aparelhos para usos específicos). } \\
\text { B03C: separação magnética ou eletrostática de materiais sólidos dos materiais sólidos ou fluidos; } \\
\text { - } \quad \text { C02Faração por meio de campos elétricos de alta-tensão. } \\
\text { - } \quad \text { C08G: composto de água, de águas residuais, de esgotos ou de lamas e lodos. } \\
\text { insaturadas carbono-carbono (processos de fermentação ou processos que utilizem enzimas para } \\
\text { sintetizar uma composição ou composto químico desejado ou para separar isômeros ópticos de } \\
\text { uma mistura racência). } \\
\text { C08K: uso de substâncias inorgânicas ou orgânicas não-macromoleculares como ingredientes de } \\
\text { composições (tintas para pinturas, tintas de escrever, vernizes, corantes, produtos para polir, } \\
\text { adesivos). } \\
\text { C08L: composições de compostos macromoleculares (composições baseadas nos monômeros } \\
\text { polimerizáveis; filamentos ou fibras artificiais; composições para o tratamento de têxteis). } \\
\text { C09D: composições de revestimento, p. ex. tintas, vernizes ou lacas; pastas de enchimento; } \\
\text { removedores químicos de tintas para pintar ou imprimir; tintas para imprimir; líquidos corretivos; } \\
\text { corantes para madeira; pastas ou sólidos para colorir ou imprimir; uso de materiais para esse fim } \\
\text { (cosméticosa; processos para aplicar líquidos ou outros materiais fluentes a superfícies em geral; } \\
\text { coloração da madeira; vidrados ou esmaltes vítreos; resinas naturais, verniz a álcool, óleos } \\
\text { secantes, secantes (sedativos), terebintina; composições polidoras que não o verniz à álcool, ceras } \\
\text { para esquis; adesivos ou uso de materiais como adesivos; materiais for vedação ou empanque de } \\
\text { juntas ou tampas; materiais for vedação de vazamentos; processos para a produção eletrolítica ou } \\
\text { eletroforética de revestimentos. }\end{array}$ \\
\hline
\end{tabular}

Fonte: Dados da pesquisa.

A Universidade Federal da Bahia se insere na rede tecnológica com a Petrobras na área G01N/023, que corresponde a tecnologias de análise de materiais pelo uso da radiação de ondas ou partículas. A Universidade Federal do Pará, está desenvolvendo tecnologias na área $\mathrm{C} 05 \mathrm{G}$, para a produção de fertilizantes. Em relação à Universidade Federal de Santa Catarina, a classe é a F28D, refere-se ao 
desenvolvimento de materiais para transferência, troca ou armazenamento de calor, como aquecedores de fluidos dotados de meios geradores de calor e de meios de transferência de calor. Já a Universidade Federal de Santa Maria está na rede com o desenvolvimento de tecnologias para separar materiais, por exemplo, separação de sólidos de outros sólidos.

\section{CONCLUSÃO}

O estudo conclui que a Petrobrás, diante do cenário de incentivos e políticas (como a obrigatoriedade de investir $1 \%$ do seu lucro bruto em pesquisas) contribui de modo significativo para o desenvolvimento dos NITs no âmbito das universidades. Isto determinou a necessidade de modificações que implicaram investimentos e relações intersetoriais por parte das distintas unidades de pesquisa, como a criação das redes tecnológicas de pesquisa, evidenciadas através da análise da rede de cooperação tecnológica.

A principal conclusão obtida é que as universidades que se inseriram nas redes de cooperação tecnológica com a Estatal estão com avanços significativos no desenvolvimento de inovações. No ramo do petróleo, como mostra a rede a UFRJ com tecnologias para exploração e produção e no desenvolvimento de novos materiais para produtos a partir da química verde (como óleo para cosméticos) e para o desenvolvimento da bioenergia, como é o caso da rede da USP e UNICAMP.

Os fatores que influenciaram a formação das redes tecnológicas com as universidades também podem ser explicados a partir da obrigatoriedade da Petrobras de direcionar parte dos seus lucros com pesquisas, em especial, para as universidades. Foi nesse período que ocorreu um salto de infraestrutura de pesquisa (como mostra o relatório do IPEA) e no desenvolvimento de novas frentes tecnológicas no país, que possivelmente, permitiram que invenções fossem agora regulamentadas em um regime de propriedade intelectual nas universidades.

A concepção de inovação tecnológica torna-se cada vez mais sistêmica, sequencial, configurando sistemas complexos modulares, em que gargalos para a viabilidade da inovação aparecem e ao mesmo tempo surgem novos produtos, onde os processos de natureza incremental estão acoplados aos processos de inovações radicais na forma de redes de pesquisa, que recriam na atualidade as parcerias e as novas formas de acordos contratuais. O presente trabalho contribui para entendimento deste processo e dos agentes envolvidos nos processos, no âmbito da Petrobras, e das áreas de cooperação entre eles. O desafio é continuar analisando a formação dessas redes tecnológicas em relação e a cooperação entre instituições públicas com os setores industriais privados.

\section{REFERÊNCIAS}

ALTBACH, P. Empires of Knowledge and Development, In Altbach, P.; Balán, J. (eds), World Class Worldwide: Transforming Research Universities in Asia and Latin America, The Johns Hopkins University Press: Baltimore, 2007.

ACHTERT, E.; BÖHM, C.; KRÖGER, P. (2006). "DeLi-Clu: Boosting Robustness, Completeness, Usability, and Efficiency of Hierarchical Clustering by a Closest Pair Ranking". LNCS: Advances in Knowledge Discovery and Data Mining. Lecture Notes in Computer Science 3918: 119-128.

Base Derwent Innovations Index, available at <http://www.periodicos.capes.gov.br/>.

BUENO, Carolina da Silveira. Cumulatividade científica e apropriabilidade do conhecimento: redes de colaboração internacional e o caso brasileiro no paradigma dos biocombustíveis. Campinas, SP: [s.n.], 2016. Disponível em: 〈http://www.bibliotecadigital.unicamp.br/document/?code=000968340>. Acesso em: 02 jun. 2016.

DAL POZ, M. E.; SILVEIRA, J. M. F. J. da. Trajetórias tecnológicas do bioetanol de segunda geração. In: Sérgio Salles-Filho (Org.). Futuros do bioetanol: o Brasil na liderança? 1ed. Rio de Janeiro/RJ: Editora Campus-Elsevier, v. 1, 2015.

DAL-POZ, Maria Ester; DA SILVEIRA, José Maria. BioEnergy Brazilian Program (BIOEN) Innovation Networks. In: BOOK OF ABSTRACTS. 2011. p. 66. 
DOSI, G. Mudança técnica e transformação industrial: a teoria e uma aplicação à indústria dos semicondutores. Campinas (SP): Editora UNICAMP, 2006 [1984]. 460p.

DOSI, G. Technological paradigms and technological trajectories: a suggested interpretation of the determinants and directions of technological change. Research Policy, Amsterdam, v. 11, n. 3, p. 147$162,1982$.

DOSI, G. MAZZUCATO, M. Knowledge Accumulation and industry evolution. The case of PharmaBiotech. Cambridge University Press, P.1-19. 2006.

EASLEY, David; KLEINBERG, Jon. Networks, crowds, and markets: Reasoning about a highly connected world. Cambridge University Press, 2010.

EPICOCO, M. Knowledge patterns and sources of leadership: mapping the semiconductor miniaturization trajectory. Research Policy, v. 42, n. 1, p.180-195, Feb. 2013.

ETZKOWITZ, H; LEYDESDORFF, L. The dynamics of innovation: from National Systems and "Mode 2" to a Triple Helix of university-industry-government relations. Research policy, v. 29, n. 2, p. 109$123,2000$.

DE NEGRI, Fernanda; SQUEFF, Flávia de Holanda Schmidt. Infraestrutura científica e tecnológica no Brasil: análises preliminares. 2014.

FUCK, Marcos Paulo et al. P\&D de interesse público? Observações a partir do estudo da Embrapa e da Petrobras. Seminário Latino-Iberoamericano de Gestion Tecnológica-ALTEC, v. 12, 2007.

FURUBOTN, E.G. - RICHTER, R. Institutions and Economic Theory. The Contribution of the New Institutional Economics. The University of Michigan Press, Ann Arbor. 1998.

GOYAL, SANJEEV. Connections: An introduction to the Economics of Networks, 2007. Princeton and Oxford: Princeton University Press.

IPC STATS, available at <http://web2.wipo.int/ipcpub/search/stats/\#version=20140101\&lang=en> .

Accesso em: Nov. 2015.

JACKSON, M. O. Social and Economic Networks, Princeton University Press. 2010.

KRAFFT, J.; QUATRARO, F.; SAVIOTTI, P. The evolution of knowledge base in knowledge-intensive sectors: social network analysis of biotechnology. Working Paper Series, n. 9, 2009.

LEYDESDORFF, L., WAGNER, C. S. International collaboration in science and the formation of a core group. JournalofInformetrics, 2(4), 2008.

MURAKAMI, T. et al. APPLYING ENTROPY INDEXES TO IDENTIFY TECHNOLOGICAL TRAJECTORIES: SECOND-GENERATION BIOETHANOL PRODUCTION. ANPEC-Associação Nacional dos Centros de Pósgraduação em Economia [Brazilian Association of Graduate Programs in Economics], 2016.

NEWMAN, M. The structure and function of complex networks. Volume 45, pages 167-256. SIAM Review. 2003.

SIMS, R.; TAYLOR, M.; SADDLER, J.; MABEE, W. From $1^{\text {st }}$ to $2^{\text {nd }}$ generation biofuel technologies: an overview of current industry and RD\&D activities. Paris: International Energy Agency (IEA), Nov. 2008. 124p. Available at <http://www.ieabioenergy.com/MediaItem.aspx?id=6060>. Acesso em: Dez. 2015.

SOUZA, L.G.A. et al. Collaborative Networks as a measure of the Innovation Systems in secondgeneration ethanol. Scientometrics (2015) 103: 355. doi:10.1007/s11192-015-1553-2.

VENTURA, V.; FRISIO, D.; FERRAZZI, G.; SILETTI, E. Forecasting the evolution of agbiotech innovation: lessons from patent data. Anais..., 17th ICABR Conference, Italy, June 2013.

WORLD INTELLECTUAL PROPERTY ORGANIZATION (WIPO), disponível em:

<http://www.wipo.int/classifications/ipc/en/>. Acesso em: Jan. 2016.

ZILBERMAN, D. The Economics of Sustainable Development. Amer. J. Agr. Econ. 96(2): 385-396; doi: 10.1093/ajae/aat075, 2013.

WU, Xindong et al. Data mining with big data. IEEE transactions on knowledge and data. 